\title{
Image Splicing Detection Based on Statistical Properties of Benford Model
}

\author{
Senfeng TONG ${ }^{1}$; Zhen $\mathrm{ZHANG}^{1}$; Yongjie XIE ${ }^{1}$; Xiaodi WU ${ }^{1}$ \\ ${ }^{1}$ School of Electrical Engineering, Zhengzhou University \\ Zhengzhou, China \\ tongsenfeng@126.com
}

\begin{abstract}
For the operation of the image splicing tamper, this paper proposes a novel approach for blind image splicing detection based on statistical properties of the Benford model. Having made discrete wavelet transform (DWT) to the test image, we extracted alternating current (AC) coefficients in discrete cosine transform (DCT) domain of the three RGB color channels from each wavelet component, and then we calculated probability distribution of the most significant digit (MSD) of the AC coefficients in DCT domain by using the Benford model, constructed the detection model of the proposed algorithm. The threshold is set according to significant level of the statistical property difference tampered between before and after, then judgement the authenticity of the images. Experimental results show that the proposed algorithm is capable to detect splicing image efficiently.
\end{abstract}

Keywords-image splicing detection; statistical characteristics; AC coefficient; most significant digit; authenticity

\section{INTRODUCTION}

Nowadays digital image has been widely used in many fields. With many powerful image processing tools, nonprofessional users can easily modify the image's content and not leave visible traces. As a consequence, people are no longer convinced of image authenticity as before. Effective methods are needed urgently to detect image forgery.

Image splicing, herein defined as a cut-and-paste of image regions from one image onto the same or another image without post-processing, is the basic operation for creating a digital photomontage. The splicing is often regarded as the first steps of image tampering; therefore image splicing has received much attention in recent years. Many researchers used statistical inconsistency to detect image splicing. Popescu et $\mathrm{al}^{[1]}$ study resampling to detect image tampering. Bayram et al applies Fourier Mellin Transform (FMT) and 1-D projection of log-polar values in their robust scheme of detecting image forgeries ${ }^{[2]}$. Johnson et al exposes digital forgeries using Chromatic Aberration ${ }^{[3]}$. Statistical moments and Markov transition probability matrix $^{[4]}$, illuminant color inconsistency ${ }^{[5]}$ and Hilbert-Huang transform and wavelet decomposition ${ }^{[6]}$ were used to detect spliced image.

In this paper, we introduce a new approach based on statistical properties of the Benford model to detect spliced images. The rest of the paper is organized as follows: In Section 2, we introduce the background of the statistic characteristics of natural images, DWT, AC coefficient and Benford's Law. Section 3 illustrates our proposed image splicing detection method, followed by our experimental results and discussion in Section 4. This is followed by conclusion and future work in Section 5.

\section{BACKGROUND}

\section{A. The statistic characteristics of natural images}

Digital images can be regarded as a two-dimensional array. Since they are derived from the real scene of the nature and obtained by digital imaging equipment, each pixels of the image has a certain degree of relevance and consistency in statistical characteristics. Image stitching and tampering operation can be achieved easily by copying and pasting. For the reason that there are other images' fragments in the spliced image, which results in inconsistency of smoothness, continuity, regularity and periodicity among image pixels, the intrinsic link among image pixels is changed and the correlation among them is reduced. The statistical characteristics also change in stitching edge even in the same image's splicing operation. The change of this relevance caused by splicing operation can be reflected through a number of statistical properties.

\section{B. Discrete wavelet transform}

DWT decomposes an image into a power of single resolution levels by applying a collection of low-pass and high-pass filters onto the image in vertical and horizontal directions. This resolution level consists of four sub-bands, which are Low Low (LL), Low High (LH), High Low (HL) and High High (HH). The LL sub-band contains most energy and therefore can be decomposed further ${ }^{[8]}$. The rest of the sub-bands contain edge details. In this paper we used onedimensional (1-D) Haar DWT, as the most possible to reduce destruction of the statistical characteristics between pixels.

\section{AC coefficient in DCT domain}

Discrete cosine transform is an orthogonal mathematical transformation closely related with Fourier transform, which has feartures of fast calculating speed and easy to implement. In this paper we used $8 \times 8$ block-discrete cosine transform ${ }^{[7]}$ (BDCT), the equation as follows:

$$
\begin{aligned}
F(k, l)= & \frac{2}{\sqrt{M N}} \sum_{k=0}^{M-1} \sum_{l=0}^{N-1} f(i, j) \\
& A(k) A(l) \cos \frac{(2 i+1) k \pi}{2 M} \cos \frac{(2 j+1) l \pi}{2 N}
\end{aligned}
$$


In equation (1) where $A(k)=\left\{\begin{array}{c}\frac{1}{\sqrt{2}}, k=0 \\ 1, k=1, \cdots, M-1\end{array}\right.$,

$$
A(l)=\left\{\begin{array}{c}
\frac{1}{\sqrt{2}}, l=0 \\
1, l=1, \cdots, N-1
\end{array}\right.
$$

After DCT transformation,one DC component (DC coefficient) and sixty-three AC components (AC coefficient) can be obtained,where DC coefficients represent pixel density mean value of block,the low frequencies AC coefficients contain rich image texture information and the high frequencies AC coefficients contain some details of the image.

\section{Benford's Law}

A statistical law called Benford's Law, was introduced by Frank Benford in 1938 and predicts the frequency of appearance of the MSD for a broad range of natural and artificial data. A typical probability distribution of Benford's Law is shown in Figure 1.

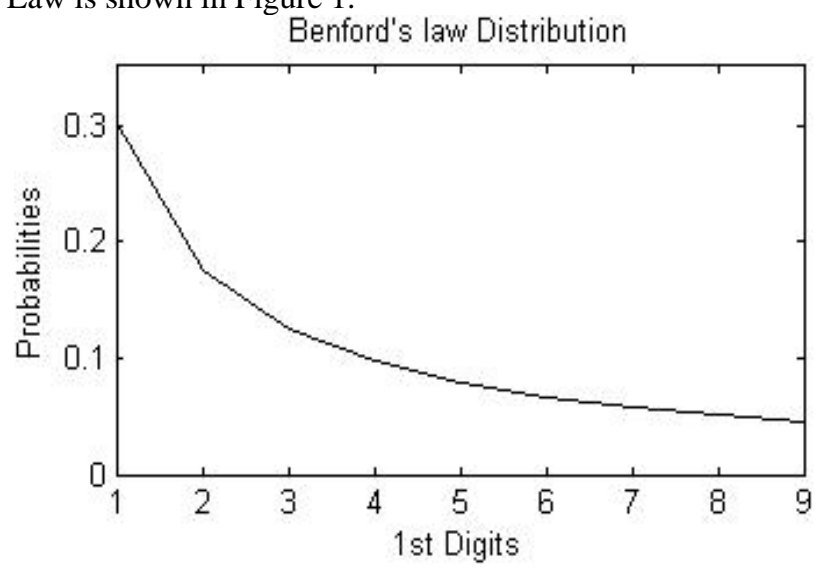

Figure 1. Probability Distribution of Benford's Law

In recent years it has attracted the attention of image processing experts. In 2005, Acebo et $\mathrm{al}^{[9]}$, showed how light intensity in an image can be used to determine if an image is genuine or computer generated. Similarly, Fu et al ${ }^{[10]}$ have applied Benford's Law on DCT coefficients in order to detect unknown JPEG compression. Unlike reference 9, we have applied DWT before calculating 1st digit probabilities and analysed images in the frequency domain, which is better to separate edge details from low frequencies. The distribution for Benford's Law can be expressed by equation as follows:

$$
P(x)=\log _{10}\left(1+\frac{1}{x}\right) \quad x=1,2 \ldots 9
$$

Where $\mathrm{x}$ is the first digits of the number and $\mathrm{P}(\mathrm{x})$ is the probability distribution of $\mathrm{x}$.

\section{Proposed Method}

\section{A. The Statistic Characteristics of DCT Domain}

Given a number in decimal form, the most significant digit (MSD) is simply the leading digit of the mantissa (assuming that the exponent is a power of 10); hence, the MSD cannot take the value 0 . For instance, the MSD of 3.21 is 3, and the MSD of 0.00431 is 4 . From DCT domain's statistical properties proposed by reference [10], we find that statistical properties of natural images' DCT coefficients are not strictly stable. Statistics show that probability distribution of the MSD between different images' DCT coefficients have some difference, even the probability distribution is from the same image's three color channels. A large number of experiments show that the DCT domain statistical property between spliced images and natural images have large differences. In this paper, we used Benford's Law calculate probability distribution of DCT coefficients' MSD as statistical characteristics. Figure 2 (a) is the Apple image, and (b) is the apple's MSD probability distribution of each color channel's DCT coefficients.
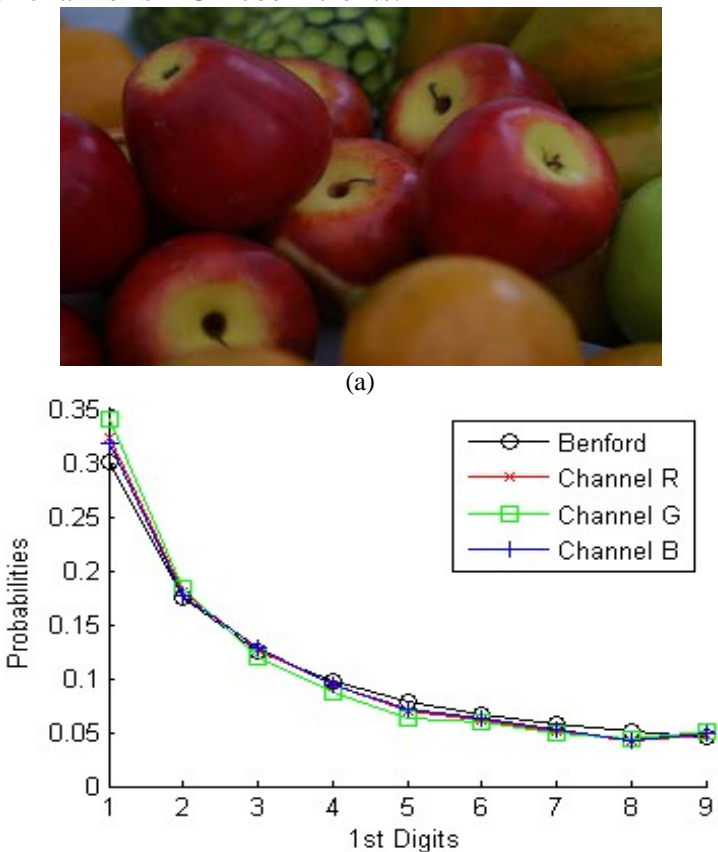

(b)

Figure 2. (a) Apple, (b) The apple's MSD probability distribution of each color channel's DCT coefficients \& Benford's Law

\section{B. Feature extraction}

By analysising image splicing, we find that splicing operation of the natural image will change the intrinsic link between the image pixels and then reduce its correlation. We also find that DCT coefficients' MSD can characterize these changes. After DCT transformation, the low frequencies alternating current (AC) coefficients contain rich image texture and edge, while the high frequencies AC coefficients contain some details of the image. The low frequencies sub- 
band of DWT mentioned above contains most of the energy and statistical characteristics of the image, while the high frequencies sub-bands contain edge details. Combined with the features of DWT and DCT, we extracted the low and high frequencies' DCT coefficients from wavelet's low frequencies sub-band while extracted the high frequencies' DCT coefficients from high frequencies sub-bands. We transformed the test image by one-dimensional (1-D) Haar DWT firstly, and then calculated DCT coefficients' MSD distribution curve and correlation's degree of each wavelet component's three RGB color channels.

Therefore, we used the average absolute difference, mean and standard deviation to calculate image's characteristic quantity. The specific method is as follows:

Definition 1. Calculate MSD's absolute difference $\overline{A D}$ of each wavelet component's three RGB color channels, the equation is as follow:

$$
\overline{A D}=\frac{1}{27} \sum_{i=1}^{9}\left(\left|P_{R_{i}}-P_{G_{i}}\right|+\left|P_{R_{i}}-P_{B_{i}}\right|+\left|P_{G_{i}}-P_{B_{i}}\right|\right)
$$

In equation (3), where $i$ is the MSD, and $i \in[1,9]$. Where $R_{i}, G_{i}, B_{i}$ is the $M S D_{i}$ of three RGB color channels’ DCT coefficients.

Definition 2. Calculate the four wavelet components absolute differences of DCT coefficients' mean $\left(M_{n}\right)$ and standard deviation ( $S$ ), the equations are as follows:

$$
\begin{gathered}
M_{n}=\frac{1}{n} \sum_{i=1}^{4} x(n) \\
S=\sqrt{E\left(X(n)-M_{n}\right)^{2}}
\end{gathered}
$$

Where $n$ is the wavelet components, and $n \in[1,4]$.

\section{Threshold Selection}

Use maximum - variance algorithm in all classes to select threshold $(\mathrm{T})$. The images will be divided into two types: spliced images A and authentic images B, A and B are two types of gap significantly. Suppose that the pixel of gray $\omega_{A}$ and the pixel of gray $\omega_{B}$ belong to $A$ and $B$ respectively, the probability distribution of $\mathrm{A}$ and $\mathrm{B}$ are as follows:

$$
\omega_{A}=\omega(T)=\sum_{i=1}^{T} P_{i}, \omega_{B}=1-\omega(T)
$$

The average gray degree of A and B can respectively as follows:

$$
\mu_{A}=\frac{\mu(T)}{\omega(T)}, \mu_{B}=\frac{\mu-\mu(T)}{1-\omega(T)}
$$

Where is the average gray degree of all images:

$$
\mu=\sum_{i=1}^{T} i^{*} P_{i}
$$

The variance of class $\mathrm{A}$ and $\mathrm{B}$ as follows:

$$
\sigma^{2}=\omega(T)\left(\mu_{A}-\mu\right)^{2}+(1-\omega(T))\left(\mu_{B}-\mu\right)^{2}
$$

So the $\mathrm{T}$ is the desired value to make the maximum variance of class $A$ and $B$.

\section{Proposed Method flow chart}

The algorithm process is shown in figure 3; the test image is first transformed by 1-D Haar DWT. Each color channel of each wavelet sub-band is divided into nonoverlapping sub-blocks of $8 \times 8$ pixels and DCT is applied to each block, and the probability distribution of each color channel's MSD of three DWT coefficients is obtained by using the Benford's Law model. Then we will received three probability distribution curves of each wavelet sub-band, and calculated theirs absolute difference and variance. Finally, we calculated mean and standard deviation of four subbands' absolute difference. If the standard deviation (S) is greater than the threshold $(\mathrm{T})$ of experiment measured, the test image will judge as authentic image, else will judge as spliced image.

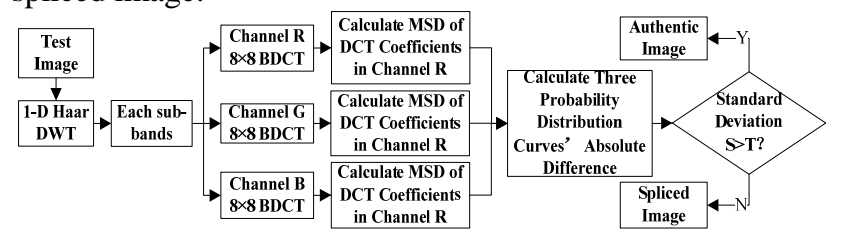

Figure 3. The flow chart of splicing detection algorithm

\section{EXPERIMENTAL RESULTS AND DISCUSSION}

In this paper we used Columbia Uncompressed Image Splicing Detection Evaluation Dataset ${ }^{[11]}$ as experimental data. This dataset has 363 uncompressed TIFF format or BMP format images, 183 of them are authentic images, and 180 are spliced ones. The authentic images are taken with our four digital cameras, while splicing images are created the spliced images from the other authentic image set using Adobe Photoshop. In order to focus only on the effects of splicing, no post processing was performed.

We have implemented the detection method in Matlab $\mathrm{R} 2011 \mathrm{~b}$, and the experimental environment is on a personal computer of $1.6 \mathrm{GHz}$ processors with 2GB memory. We choose 100 authentic images and 100 spliced images, the statistical results of each image's standard deviation (S) as shown Figure 4.

\section{CONCLUSION AND FUTURE WORK}

We proposed a novel method for splicing image detection in this paper. The detection was based on statistical properties of the Benford model. We have constructed a statistical detection model for the distribution of the first digits of the DCT coefficients. Experimental results showed that this approach is indeed effective in detecting spliced images. Our detection algorithm aims at spliced from different image, future research we will focus on the spliced from same image and the location of tamper areas.

\section{ACKNOWLEDGMENT}

This paper is supported in part by Provincial Major Scientific and Technological Research Projects Grant Nos. 092101210100. 


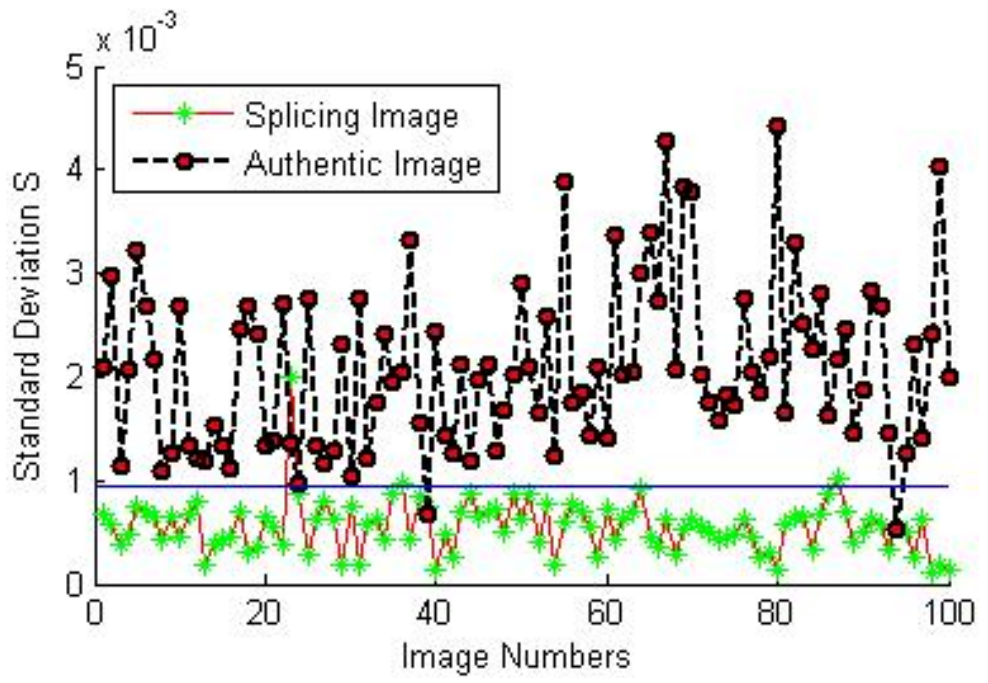

Figure 4. The statistical results of experiment images' standard deviation S

TABLE I. The ReSUlt OF DETECTION IMAGE DATASET

\begin{tabular}{|c|c|c|c|c|c|c|c|c|}
\hline \multirow{2}{*}{$\begin{array}{l}\text { Camera } \\
\text { Model }\end{array}$} & \multirow{2}{*}{$\begin{array}{c}\text { Number of } \\
\text { authentic } \\
\text { image }\end{array}$} & \multirow{2}{*}{$\begin{array}{c}\text { Spliced camera } \\
\text { model }\end{array}$} & \multirow{2}{*}{$\begin{array}{c}\text { Number of } \\
\text { spliced image }\end{array}$} & \multicolumn{5}{|c|}{ Judgment accuracy under different threshold } \\
\hline & & & & 0.00086 & 0.0009 & 0.00094 & 0.001 & 0.00104 \\
\hline \multirow{3}{*}{ Canon G3 } & \multirow{3}{*}{27} & Nikon D70 & 30 & \multirow{3}{*}{$91.46 \%$} & \multirow{3}{*}{$95.73 \%$} & \multirow{3}{*}{$98.30 \%$} & \multirow{3}{*}{$97.44 \%$} & \multirow{3}{*}{$93.16 \%$} \\
\hline & & $\begin{array}{c}\text { Canon 350D Rebel } \\
\text { XT }\end{array}$ & 30 & & & & & \\
\hline & & Kodak DCS 330 & 30 & & & & & \\
\hline \multirow[t]{2}{*}{ Nikon D70 } & \multirow[t]{2}{*}{117} & $\begin{array}{c}\text { Canon 350D Rebel } \\
\text { XT }\end{array}$ & 30 & \multirow[t]{2}{*}{$91.53 \%$} & \multirow[t]{2}{*}{$96.62 \%$} & \multirow[t]{2}{*}{$97.75 \%$} & \multirow[t]{2}{*}{$98.87 \%$} & \multirow[t]{2}{*}{$94.35 \%$} \\
\hline & & Kodak DCS 330 & 30 & & & & & \\
\hline $\begin{array}{c}\text { Canon 350D } \\
\text { Rebel XT }\end{array}$ & 36 & Kodak DCS 330 & 30 & $87.88 \%$ & $95.45 \%$ & $96.96 \%$ & $93.94 \%$ & $89.40 \%$ \\
\hline
\end{tabular}

\section{REFERENCES}

[1] A. C. Popescu and H. Fraid, "Exposing Digital Forgeries by Detecting Traces of Resamping”, IEEE Transactions on Signal Processing, Vol. 53, pp.758-767, 2005.

[2] S. Bayram, T. Sencar, N. Memon, “An Efficient and Robust Method for Detecting Copy-move Forgery”, ICASSP 2009, pp.1053-1056.

[3] M. K. Johnson, H. Farid, "Exposing Digital Foorgeries Through Chromatic Aberration", in Processings of the 8th workshop on Multimedia and Security, pp.48-55, 2006.

[4] Y. Q. Shi, C. H. Chen, W. Chen, "A natural image model approach to splicing detection", Proceedings of the 9th workshop on Multimedia \& security, pp.51-62, 2007.

[5] D. Fu, Y. Q. Shi, W. Su, "Detection of image splicing based on Hilbert-Huang transform and moments of characteristic functions with wavelet decomposition”, Proceeding of 5th International Workshop on Digital Watermarking, pp.177-187, 2006.

[6] X. M. Wu, Z. Feng, "Image Splicing Detection Using Illuminant Color Inconsistency”, 2011 Third International Conference on Multimedia Information Networking and Security, pp.600-603, 2011.
[7] M. Ghorbani, M. Firouzmand, A. Faraahi, "DWT-DCT (QCD) Based Copy-move Image Forgery Detection”, Systems, 2011 18th International Conference on Signals and Image Processing, pp.1-4, 2011.

[8] H. Farid, S. W. Lyu, "Higher-order Wavelet Statistics and their Application to Digital Forensics", Proceedings of the 2003 Conference on Computer Vision and Pattern Recognition Workshop, pp.1-8, 2003.

[9] E. D. Acebo, M. Sbert, "Benford's law for natural and synthetic images", Workshop Computational Aesthetics in Graphics, Visualization, and Imaging, pp.169-176, 2005.

[10] D. Fu, Y. Q. Shi, W. Su (eds.), “A Generalized Benford`s Law for JPEG Coefficients and Its Applications in Image Forensics”, SPIE Electronic Imaging, 2007.

[11] Y. F. Hsu, S. F.Chang, "Detecting Image Splicing using Geometry Invariants and Camera Characteristics Consistency”, International Conference on Multimedia and Expo, pp.549-552, 2006. 